10,11

\title{
Кислородные вакансии и валентные состояния железа в соединениях $\mathrm{SrFeO}_{3-\delta}$
}

\author{
() В.Д. Седых, О.Г. Рыбченко, Э.В. Суворов, А.И. Иванов, В.И. Кулаков
}

Институт физики твердого тела РАН,

Черноголовка, Россия

E-mail: sedykh@issp.ac.ru

Поступила в Редакцию 22 апреля 2020 г.

В окончательной редакции 22 апреля 2020 г.

Принята к публикации 25 мая 2020 г.

\begin{abstract}
Проведены рентгеновские и мёссбауэровские исследования браунмиллеритной фазы и проанализированы известные в литературе данные по однофазным соединениям с перовскитной структурой в феррите стронция $\mathrm{SrFeO}_{3-\delta}$. Установлено, что все валентные состояния железа для любого фазового состава феррита стронция определяются его локальным кислородным окружением. Это позволяет понять закономерность переходов железа из одного валентного состояния в другое при добавлении кислородных вакансий и объяснить структурные состояния железа в феррите стронция, включающие как однофазные, так и двухфазные составы. Такой подход является более общим случаем для описания всех известных соединений и синтезируемых комбинаций фаз в $\mathrm{SrFeO}_{3-\delta}$, чем рассматриваемая в литературе формула для однофазных структур, которая хорошо встраивается в предложенную схему.
\end{abstract}

Ключевые слова: феррит стронция; рентгеновские спектры; мёссбауэровские спектры; перовскиты, кислородные вакансии.

DOI: 10.21883/FTT.2020.10.49924.096

\section{1. Введение}

Перовскитные твердые растворы с общей формулой $\mathrm{A}_{1-x} \mathrm{Sr}_{x} \mathrm{FeO}_{3-\delta}\left(\mathrm{A}=\mathrm{La}^{3+}, \mathrm{Bi}^{3+}, \mathrm{Ba}^{2+}\right)$ являются перспективными объектами в качестве катодных материалов в твердооксидных топливных элементах (ТОТЭ) и мембран кислородного просачивания [1-3]. Стронциевый феррит $\mathrm{SrFeO}_{3-\delta}$ является одним из исходных материалов для этих твердых растворов и для исследования свойств сложных катодных материалов необходимо в первую очередь его тщательное изучение. Композиции материала сильно зависят от содержания кислорода. Согласно литературным данным [4-10], в кислороднодефицитном феррите стронция $\mathrm{SrFeO}_{3-\delta}$ существуют четыре однофазные структуры: $\mathrm{SrFeO}_{3}$ (кубическая сингония), $\mathrm{SrFeO}_{2.86}$ (тетрагональная), $\mathrm{SrFeO}_{2.73}$ (ромбическая) и $\mathrm{SrFeO}_{2.50}$ (ромбическая, тип браунмиллерит). Эти однофазные структуры описываются общей схемой, предложенной в работе [4]: $\mathrm{SrFeO}_{3-1 / n}(n=\infty, 8,4,2)$, где на одну вакансию приходится $\mathrm{n}$ исходных перовскитных ячеек. Экспериментально получаемые фазы близки по содержанию кислорода к идеальным однофазным композициям - $\mathrm{SrFeO}_{3}(n \rightarrow \infty), \mathrm{Sr}_{8} \mathrm{Fe}_{8} \mathrm{O}_{23}$ (или $\mathrm{SrFeO}_{2.875}$, $n=8$ ), $\mathrm{Sr}_{4} \mathrm{Fe}_{4} \mathrm{O}_{11}$ (или $\left.\mathrm{SrFeO}_{2.75}, n=4\right), \mathrm{Sr}_{2} \mathrm{Fe}_{2} \mathrm{O}_{5}$ (или $\left.\mathrm{SrFeO}_{2.50}, n=2\right)$. При $n \rightarrow \infty$ соединение имеет стехиометрический состав по кислороду (без вакансий) и содержит только катионы $\mathrm{Fe}^{4+}[4,5]$. При $n=2$ соединение содержит только катионы $\mathrm{Fe}^{3+}$. Промежуточные состояния имеют смешанную валентность железа.

Следует подчеркнуть, что вышеприведенная схема применима только к однофазным структурам, в которых одна вакансия приходится на $n$ перовскитных ячеек. Структуры фаз $\mathrm{Sr}_{8} \mathrm{Fe}_{8} \mathrm{O}_{23}, \mathrm{Sr}_{4} \mathrm{Fe}_{4} \mathrm{O}_{11}$ и $\mathrm{Sr}_{2} \mathrm{Fe}_{2} \mathrm{O}_{5}$ представляют собой сверхрешетки, состоящие из нескольких перовскитных ячеек. Эти структуры являются кислородно-вакансионно упорядоченными, что было показано с помощью комбинации методов рентгеновской и электронной дифракции $[4,8,11,12]$. Таким образом, есть четыре однофазные структуры с определенным набором атомов железа разной валентности с разным локальным кислородным окружением. Также из литературных данных следует, что в зависимости от структуры часть атомов Fe имеет октаэдрическое кислородное окружение $\mathrm{FeO}_{6}$, другая часть - квадратно-пирамидальное $\mathrm{FeO}_{5}$ [13-15]. Однако до сих пор нет однозначного мнения относительно того, атомы Fе какой валентности имеют то или иное кислородное окружение. Например, в работах $[7,16]$ рассматривается, что в $\mathrm{SrFeO}_{2.75}$ ионы $\mathrm{Fe}^{3+}$ имеют пирамидальное кислородное окружение, а все ионы $\mathrm{Fe}^{4+}$ - октаэдрическое. При уменьшении содержания кислорода (появлении кислородных вакансий) меняется валентное состояние железа, его окружение; при значительном изменении состава по кислороду происходят фазовые переходы.

Кроме однофазных структур в феррите стронция существует также целый набор малоизученных соединений с промежуточными составами по кислороду, которые в литературе рассматриваются как смеси, состоящие из двух фаз $[4,9,11,14,17-19]$ и вышеприведенной формулой не описываются. В то же время именно полное исследование как однофазных, так и двухфазных структурных состояний позволяет проследить характер перехода 
железа из одного валентного состояния в другое при изменении содержания кислорода, другими словами, при добавлении вакансий.

Таким образом, исходя из формулы для однофазных структур с разным кислородным содержанием, предлагаемой в литературе, невозможно проследить характер перехода железа из одного валентного состояния в другое и изменение его локального кислородного окружения при добавлении вакансий во всем кислородном интервале, включающем как однофазные, так и двухфазные составы.

Информацию обо всех возможных валентных состояниях железа, его локальных окружениях в пределах структуры и соотношении этих окружений, позволяет получать мёссбауэровская спектроскопия. Структуру, параметры кристаллической решетки, фазовый состав обычно получают из рентгеновских и нейтронографических данных.

Учитывая вышесказанное, предлагается новый подход отличный от схемы работы [4]. Основой этого подхода является рассмотрение характера изменения валентного состояния железа в оксиде $\mathrm{SrFeO}_{3-\delta}$, которое определяется количеством вакансий в кислородном интервале существования структурных состояний $(0 \leq \delta \leq 0.5)$ независимо от фазового состояния образца. Наиболее информативным и точным методом получения валентных состояний железа является мёссбауэровская спектроскопия. Предлагаемый подход позволяет не только проследить, как при изменении количества вакансий меняется валентное состояние $\mathrm{Fe}$, но также количественно оценить доли этих валентных состояний. Зная типы и количество валентных состояний в однофазных структурах, можно, используя данный подход, оценить вклады каждой фазы в двухфазных образцах. Именно в этом состоит отличие предлагаемого подхода от схемы [4]. Кроме того, знание характера изменения валентности железа при добавлении вакансий может быть полезным при изучении физических свойств, особенно аномальных, ферритса стронция. Для построения предлагаемой схемы не хватало мёсбауэровских данных для браунмиллеритной фазы и смеси браунмиллеритной и ромбической фаз. С этой целью дополнительно были получены и проведены рентгеновские и мёссбауэровские исследования браунмиллеритной фазы и смеси браунмиллеритной и ромбической фаз. Кроме того проанализированы известные литературные данные по однофазным и двухфазным образцам.

\section{2. Эксперимент}

Для получения браунмиллеритной фазы синтезированный методом золь-гель феррит стронция был отожжен в вакууме при двух температурах $-450^{\circ} \mathrm{C}$ и $650^{\circ} \mathrm{C}$. Синтез образца подробно описан в работе [20].

Структурная аттестация поликристаллических образцов проводилась при комнатной температуре на дифрак- тометре Siemens D-500 (излучение Co $K_{\alpha}$ ). Фазовый состав образцов определялся с использованием программ PowderCell 2.4 и Match 3.

Мёссбауэровские измерения выполнены при комнатной температуре на поликристаллических образцах на спектрометре СМ1101, работающем в режиме постоянного ускорения, с использованием радиоактивного источника ${ }^{57} \mathrm{Co}(\mathrm{Rh})$. При анализе мёссбауэровских спектров поглощения использовалась программа MossFit (версия 3.1) для разложения спектров на парциальные спектры путем их аппроксимации суммой аналитических функций. Алгоритмы программы соответствуют приближению тонкого поглотителя.

\section{3. Результаты и обсуждение}

\section{1. Рентгеноструктурный анализ}

Рентгенограмма феррита стронция $\mathrm{SrFeO}_{3-\delta}$, отожженного при $450^{\circ} \mathrm{C}$, приведена на рис. $1, a$. В образце присутствуют две фазы: $\mathrm{Sr}_{4} \mathrm{Fe}_{4} \mathrm{O}_{11}$, которая имеет ромбическую решетку с пространственной группой Cmmm [13] и параметрами элементарной ячейки $a=10.982 \AA$, $b=7.708 \AA, c=5.478 \AA$ и $S_{2} \mathrm{Fe}_{2} \mathrm{O}_{5}$ (или $\mathrm{SrFeO}_{2.5}$ ) со структурой браунмиллерита [21] (пр. гр. Ibm2, параметры ячейки $a=5.674 \AA, b=15.584 \AA, c=5.530 \AA)$. Количественная оценка, выполненная с помощью программы PowderCell 2.4, дает соотношение этих фаз 55:45\%.

Образец, отожженный в вакууме при температуре $650^{\circ} \mathrm{C}$ (рис. $\left.1, b\right)$, представляет собой практически чистый $\mathrm{Sr}_{2} \mathrm{Fe}_{2} \mathrm{O}_{5}$ (структура браунмиллерита, пр.гр. Ibm2, параметры ячейки $a=5.673 \AA$, $b=15.592 \AA, c=5.529 \AA$ ).

\section{2. Мёссбауэровская спектроскопия}

Мёссбауэровский спектр любого исследуемого объекта представляет собой сумму парциальных спектров, где каждый подспектр соответствует определенному локальному окружению мёссбауэровского атома. Мёссбауэровский спектр не дает прямой информации о структурном состоянии образца. Однако, зная валентные состояния железа и их долю в однофазном образце, можно из мёссбауэровских данных оценить фазовый состав для двухфазных образцов. В ферритах стронция в ближайшем окружении атомов железа находится кислород.

Мёссбауэровский спектр синтезированного образца $\mathrm{SrFeO}_{3-\delta}$, отожженного в вакууме при температуре $450^{\circ} \mathrm{C}$, показан на рис. $2, a$. Он состоит из двух магнитных подспектров, изомерные сдвиги которых соответствуют трехвалентному железу в браунмиллеритной фазе с двумя кислородными окружениями, октаэдрическим и тетраэдрическим, в соотношении 23 и $26 \%$ соответственно. Кроме того, в спектре есть парамагнитная часть (51\%), состоящая из двух подспектров, параметры которых соответствуют локальным окружениям, присущим ромбической фазе $\mathrm{Sr}_{4} \mathrm{Fe}_{4} \mathrm{O}_{11}$ [20], т.е. 


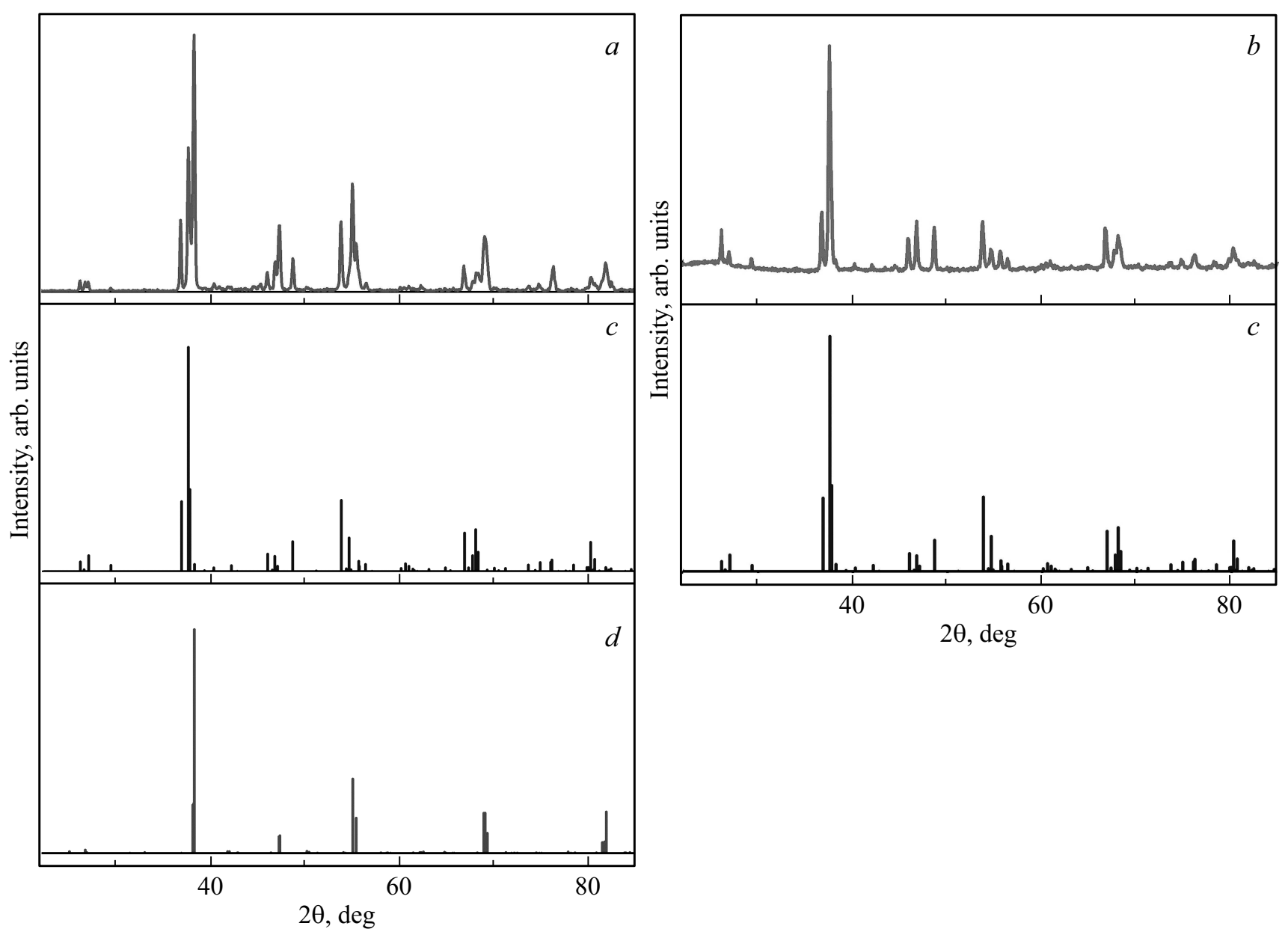

Рис. 1. Дифрактограммы образца $\mathrm{SrFeO}_{3-\delta}$, отожженного в вакууме при температурах: $a-450^{\circ} \mathrm{C}, b-650^{\circ} \mathrm{C}$; для сравнения на рисунках представлены штрих-диаграммы: $c-\mathrm{Sr}_{2} \mathrm{Fe}_{2} \mathrm{O}_{5}$ (Match! № 96-200-2241) и $d-\mathrm{Sr}_{4} \mathrm{Fe}_{4} \mathrm{O}_{11}$ (Match! № 96-152-1172).
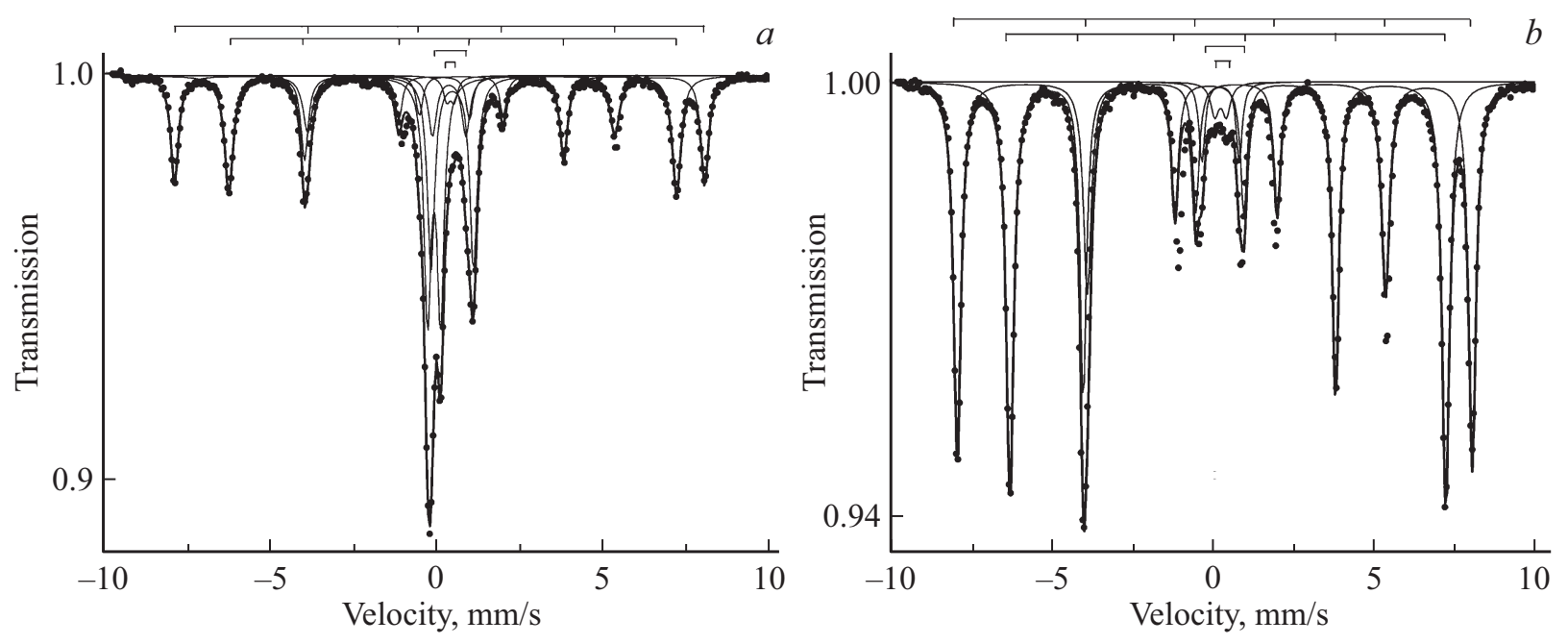

Рис. 2. Мёссбауэровские спектры, измеренные при $300 \mathrm{~K}$, для образца, отожженного в вакууме при температуре: $a-450^{\circ} \mathrm{C}$, $b-650^{\circ} \mathrm{C}$.

полученный образец двухфазный и соотношение локальных окружений для браунмиллеритной и ромбической фаз примерно равное. После вакуумного отжига при температуре $650^{\circ} \mathrm{C}$ два магнитных подспектра, соответствующие браунмиллеритной фазе, составляют 94\% (рис. $2, b$ ). Парамагнитная часть спектра соответствует 
Таблица 1. Параметры мёссбауэровских парциальных спектров при $300 \mathrm{~K}$ образца $\mathrm{SrFeO}_{3-\delta}$, отожженного в вакууме при $450^{\circ} \mathrm{C}$ $(450 \mathrm{AV})$ и $6500 \mathrm{C}(650 \mathrm{AV})$

\begin{tabular}{|c|c|c|c|c|c|c|}
\hline Annealing & Phase & Partial spectrum & $\mathrm{IS}, \mathrm{mm} / \mathrm{s}$ & $\Delta, \mathrm{mm} / \mathrm{s}$ & $H_{n}, \mathrm{kOe}$ & $\mathrm{A}, \%$ \\
\hline $\begin{array}{l}\text { 450AV } \\
650 \mathrm{AV}\end{array}$ & $\begin{array}{l}\mathrm{B} \\
\mathrm{Rh} \\
\mathrm{B} \\
\mathrm{Rh}\end{array}$ & $\begin{array}{l}\text { Sx }-1 \\
\text { Sx }-2 \\
D-1 \\
D-2 \\
\text { Sx-1 } \\
\text { Sx }-2 \\
D-1 \\
D-2\end{array}$ & $\begin{array}{r}0.38(1) \\
0.17(1) \\
-0.09(1) \\
0.42(1) \\
0.38(1) \\
0.17(1) \\
0.23(5) \\
0.24(5)\end{array}$ & $\begin{array}{r}0.66(1) \\
-0.56(1) \\
0.39(1) \\
1.28(1) \\
0.67(1) \\
-0.57(1) \\
0.36(5) \\
1.17(5)\end{array}$ & $\begin{array}{l}496(1) \\
419(1) \\
496(1) \\
420(1)\end{array}$ & $\begin{array}{r}23 \\
26 \\
24 \\
27 \\
44 \\
50 \\
2 \\
4\end{array}$ \\
\hline
\end{tabular}

Примечание. $\mathrm{Rh}-$ ромбическая фаза, B - браунмиллеритная фаза; $\mathrm{Sx}-1$ и $\mathrm{Sx}-2-$ секстеты, D-1 и D-2 - дублеты; IS - изомерный сдвиг (относительно ОЦК Fе при $300 \mathrm{~K}$ ) , $\Delta$ - квадрупольное расщепление, $H_{n}$ - эффективное магнитное поле на ядре ${ }^{57}$ Fе для разных позиций ионов железа в решетке, А - вклад данного подспектра.

остатку фазы $\mathrm{Sr}_{4} \mathrm{Fe}_{4} \mathrm{O}_{11}$. Параметры парциальных мёссбауэровских спектров для обоих образцов приведены в табл. 1. Полученные результаты хорошо согласуются с литературными данными [4,5,8,22,23].

Как следует из мёсбауэровских данных [20], во всех фазах оксида $\mathrm{SrFeO}_{3-\delta}$ суммарно существует три валентных состояния железа: $\mathrm{Fe}^{4+}, \mathrm{Fe}^{3.5+}$ и $\mathrm{Fe}^{3+}$, и четыре кислородных окружения: $\mathrm{Fe}^{4+}$ имеет два кислородных окружения - октаэдрическое $\left(\mathrm{Fe}^{4+}(\mathrm{O})\right)$ и пирамидальное $\left(\mathrm{Fe}^{4+}(\mathrm{P})\right)$, а $\mathrm{Fe}^{3.5+}(\mathrm{O})$ и $\mathrm{Fe}^{3+}(\mathrm{O})$ октаэдрическое. Кроме того, в браунмиллеритной фазе ионы $\mathrm{Fe}^{3+}$ имеют два окружения: октаэдрическое и тетраэдрическое. Каждая структурная композиция имеет свой набор валентных состояний железа с определенным кислородным окружением. С изменением содержания кислорода, точнее, с появлением кислородных вакансий, происходит переход железа из одного валентного состояния в другое, меняется их соотношение, кислородное окружение, меняется состав, часть валентных состояний исчезает, появляются другие. При этом заметные изменения содержания кислорода сопровождаются структурными превращениями. Исходя из достаточно большой величины квадрупольного расщепления для $\mathrm{Fe}^{3+}$ $(\Delta=1.2-1.3 \mathrm{~mm} / \mathrm{s})$ в конфигурации $\mathrm{Sr}_{4} \mathrm{Fe}_{4} \mathrm{O}_{11}$ можно сказать, что $\mathrm{Fe}^{3+}$ находится в искаженном октаэдрическом окружении. Если бы $\mathrm{Fe}^{3+}$ находился в пирамидальном окружении, как предполагается в $[7,16]$, то величина квадрупольного расщепления была бы намного больше, порядка $1.8 \mathrm{~mm} / \mathrm{s}$, как, например, для сверхпроводника $\mathrm{Bi}_{2} \mathrm{Sr}_{2} \mathrm{CaCu}_{2} \mathrm{O}_{8+X}$, легированного железом [24].

\section{3. Модельный подход}

Прежде чем перейти к предлагаемому подходу, рассмотрим все валентные состояния железа и его кислородное окружение в однофазных композициях, получаемые из мёссбауэровской спектроскопии. В однофазной композиции $\mathrm{SrFeO}_{3}$ (нет вакансий) имеется только одно кислородное окружение атомов железа единственной валентности: $\mathrm{Fe}^{4+}(\mathrm{O})$; в $\mathrm{Sr}_{8} \mathrm{Fe}_{8} \mathrm{O}_{23}$ (одна вакансия на
8 ячеек) - три кислородных окружения (три парциальных мёссбауэровских спектра): два для $\mathrm{Fe}^{4+}-$ $\mathrm{Fe}^{4+}(\mathrm{O})$ и $\mathrm{Fe}^{4+}(\mathrm{P})$ и $\mathrm{Fe}^{3.5+}(\mathrm{O})$ (рис. 3,a); в $\mathrm{Sr}_{4} \mathrm{Fe}_{4} \mathrm{O}_{11}$ (одна вакансия на 4 ячейки) - два кислородных окружения (два парциальных спектра): $\mathrm{Fe}^{4+}(\mathrm{P})$ и $\mathrm{Fe}^{3+}(\mathrm{O})$ (рис. 3,b) [20]; в браунмиллеритной фазе $\mathrm{Sr}_{2} \mathrm{Fe}_{2} \mathrm{O}_{5}$ (одна вакансия на 2 ячейки) $\mathrm{Fe}^{3+}$ имеет, как показано выше, два локальных окружения (два магнитных парциальных спектра), октаэдрическое и тетраэдрическое: $\mathrm{Fe}^{3+}(\mathrm{O}), \mathrm{Fe}^{3+}(\mathrm{T})$, рис. 3, d. Мёссбауэровские параметры для структур $\mathrm{SrFeO}_{2.875}$ и $\mathrm{SrFeO}_{2.75}$ приведены в [20]. На рис. $3, b$ и с кроме основных парциальных спектров есть дополнительные подспектры с очень небольшой интенсивностью. Наличие этих подспектров связано с тем, что получаемые образцы могут незначительно отличаться от идеальных композиций.

В нашей предыдущей работе [20] рассмотрен синтезированный образец $\mathrm{SrFeO}_{2.8}$, в котором имеются четыре основных локальных кислородных окружения железа, присущих фазам $\mathrm{Sr}_{8} \mathrm{Fe}_{8} \mathrm{O}_{23}$ и $\mathrm{Sr}_{4} \mathrm{Fe}_{4} \mathrm{O}_{11}$, т.е. $\mathrm{Fe}^{4+}(\mathrm{O})$, $\mathrm{Fe}^{4+}(\mathrm{P}), \mathrm{Fe}^{3.5+}(\mathrm{O})$ и1 $\mathrm{Fe}^{3+}(\mathrm{O})$ (рис. 3, $)$, которые соответствуют четырем парциальным спектрам. Мёссбауэровские параметры для этого образца приведены в [20]. Согласно литературным данным, образец с таким составом по кислороду предполагается двухфазным. Однако, рентгеновские исследования [20] показали, что образец однофазный. Его однофазность также подтверждается данными работы [9], в которой был синтезирован образец такого же состава по кислороду. Для этой структуры на основании полученного соотношения локальных окружений атомов железа была предложена еще одна однофазная композиция $\mathrm{Sr}_{16} \mathrm{Fe}_{16} \mathrm{O}_{45}$, в которой на 16 исходных перовскитных ячеек приходится 3 вакансии. Данных о кислородно-вакансионном упорядочении в этой композиции нет, промежуточный между $\mathrm{Sr}_{8} \mathrm{Fe}_{8} \mathrm{O}_{23}$ и $\mathrm{Sr}_{4} \mathrm{Fe}_{4} \mathrm{O}_{11}$ состав по кислороду может реализоваться в этой структуре как случайное распределение дополнительных вакансий. Полученная композиция не укладывается в рамки вышеприведенной общей формулы для идеальных однофазных композиций. Кроме того, 

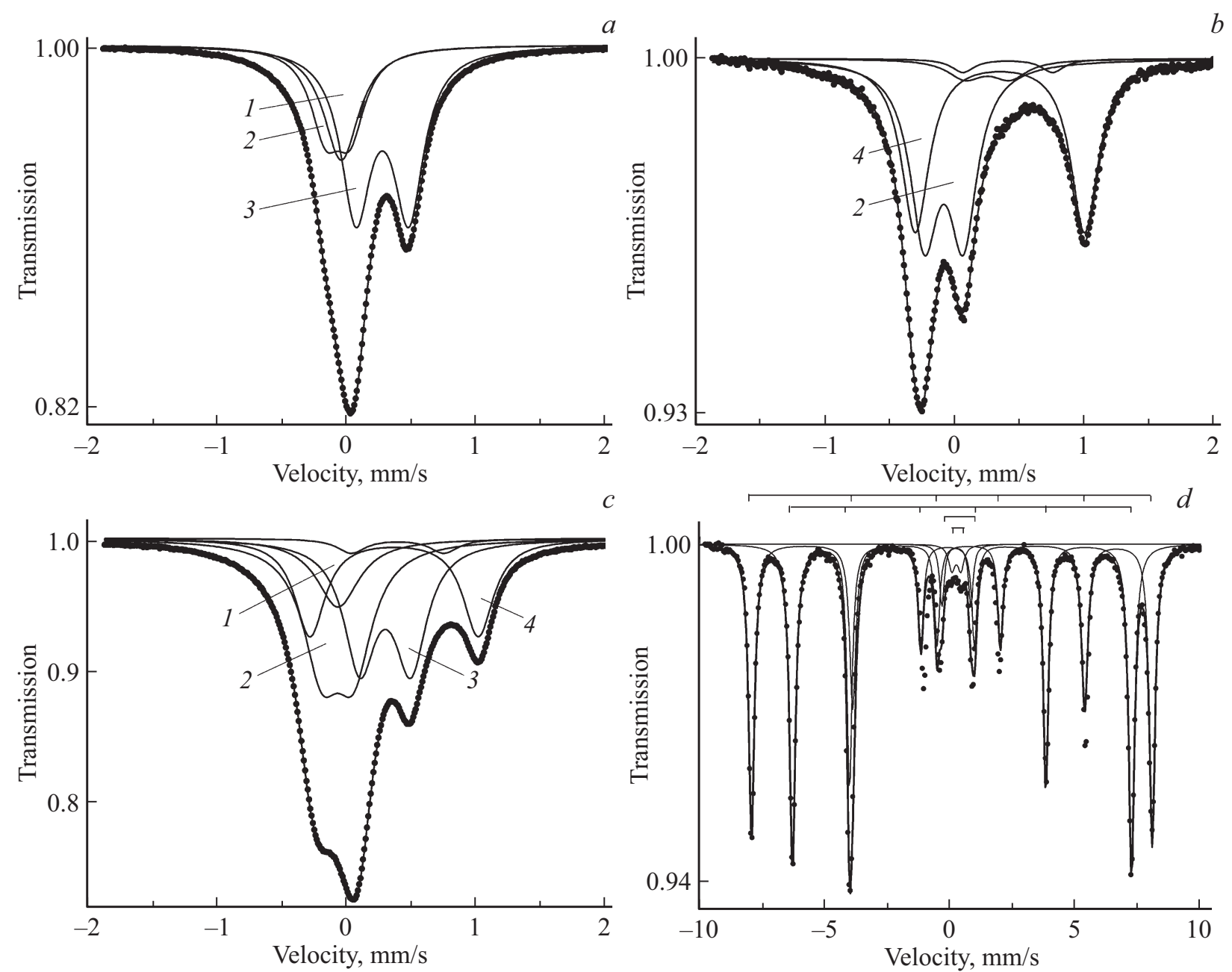

Рис. 3. Мёссбауэровские спектры, измеренные при $300 \mathrm{~K}$, образцов: $a-\mathrm{SrFeO}_{2.875}, b-\mathrm{SrFeO}_{2.75}, c-\mathrm{SrFeO}_{2.8}[15], d-6$ раунмиллерита $\mathrm{SrFeO}_{2.5}$. 1-4 - номера подспектров.

согласно литературным данным, существует также ряд двухфазных структур с разным содержанием кислорода, которые также не описываются этой формулой.

Чтобы объединить все существующие данные структурных состояний оксида $\mathrm{SrFeO}_{3-\delta}$, в настоящей работе предлагается подход, основанный на рассмотрении всех возможных локальных кислородных окружений атомов железа разной валентности в разных структурах. Такой подход дает возможность проследить, как при добавлении вакансий происходит переход железа из одного валентного состояния в другое, как при этом меняется его локальное окружение, а также оценить составы по кислороду при изменении количества вакансий.

В рамках данного подхода, исходя из мёссбауэровских данных, выбирается конфигурация с максимальным количеством перовскитных ячеек, в которой имеется весь набор валентных состояний железа с разным кислородным окружением и в которой укладываются все известные однофазные конфигурации. Этому соответствует конфигурация $\mathrm{Sr}_{16} \mathrm{Fe}_{16} \mathrm{O}_{45}$, т.е. состоящая из 16 ячеек. 16 ячеек выбирается фиксированным параметром для

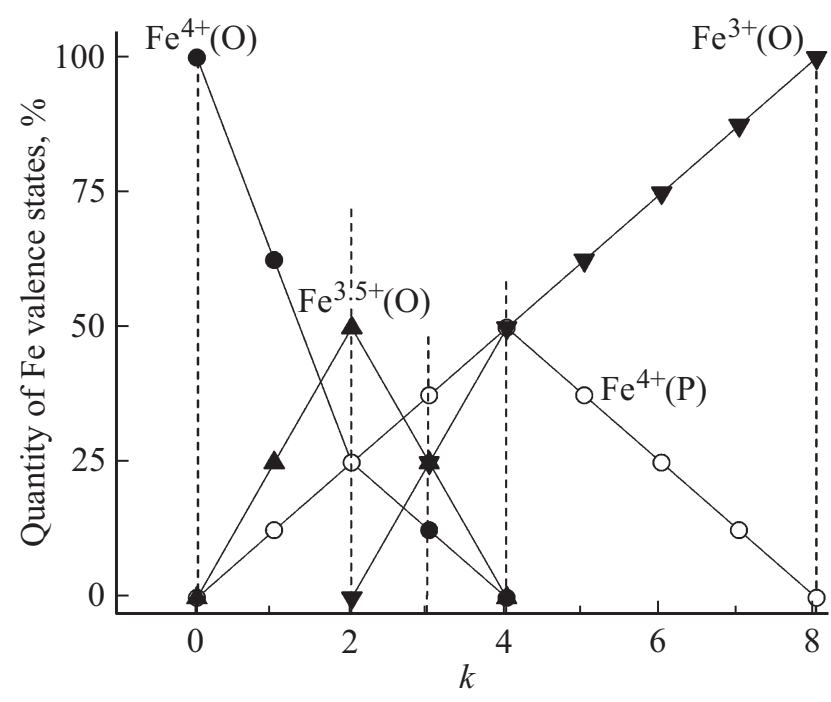

Рис. 4. Изменение количества валентных состояний $\mathrm{Fe}$ в зависимости от количества вакансий на 16 перовскитных ячеек. Вертикальные пунктирные линии показывают валентные состояния $\mathrm{Fe}$ в идеальных однофазных конфигурациях с $k=0,2,3,4,8$. 
Таблица 2. Изменение количества валентных состояний Fе в зависимости от количества вакансий в 16 перовскитных ячейках

\begin{tabular}{|c|c|c|c|c|c|c|c|}
\hline 1 & 2 & 3 & 4 & 5 & 6 & 7 & 8 \\
\hline \multirow{2}{*}{$k$} & \multirow{2}{*}{$\mathrm{SrFeO}_{3-k} / 16$} & \multirow{2}{*}{$\mathrm{SrFeO}_{3-1 / n}$} & \multicolumn{4}{|c|}{ Quantity of Fe valence state } & \multirow{2}{*}{ Phase ratio } \\
\hline & & & $\mathrm{Fe}^{4+}(\mathrm{O})$ & $\mathrm{Fe}^{4+}(\mathrm{P})$ & $\mathrm{Fe}^{3.5+}(\mathrm{O})$ & $\mathrm{Fe}^{3+}(\mathrm{O})$ & \\
\hline 0 & $\begin{array}{c}\mathrm{Sr}_{16} \mathrm{Fe}_{16} \mathrm{O}_{48} \\
\left(\mathrm{SrFeO}_{3}\right)\end{array}$ & $16\left(\mathrm{SrFeO}_{3}\right)$ & 16 & 0 & 0 & 0 & Single phase \\
\hline 1 & $\begin{array}{l}\mathrm{Sr}_{16} \mathrm{Fe}_{16} \mathrm{O}_{47} \\
\left(\mathrm{SrFeO}_{2.94}\right)\end{array}$ & $\operatorname{mix}$ & $\begin{array}{c}10 \\
(2+8)\end{array}$ & 2 & 4 & 0 & $4(\mathrm{C}) / 1$ (Tetra) \\
\hline 2 & $\begin{array}{l}\mathrm{Sr}_{16} \mathrm{Fe}_{16} \mathrm{O}_{46} \\
\left(\mathrm{SrFeO}_{2.875}\right)\end{array}$ & $2\left(\mathrm{Sr}_{8} \mathrm{Fe}_{8} \mathrm{O}_{23}\right)$ & 4 & 4 & 8 & 0 & Single phase \\
\hline 3 & $\begin{array}{l}\mathrm{Sr}_{16} \mathrm{Fe}_{16} \mathrm{O}_{45} \\
(\mathrm{SrFeO} 2.81)\end{array}$ & $\mathrm{Sr}_{16} \mathrm{Fe}_{16} \mathrm{O}_{45}$ & 2 & 6 & 4 & 4 & Single phase \\
\hline 4 & $\begin{array}{l}\mathrm{Sr}_{16} \mathrm{Fe}_{16} \mathrm{O}_{44} \\
\left(\mathrm{SrFeO}_{2.75}\right)\end{array}$ & $4\left(\mathrm{Sr}_{4} \mathrm{Fe}_{4} \mathrm{O}_{11}\right)$ & 0 & 8 & 0 & 8 & Single phase \\
\hline 5 & $\begin{array}{l}\mathrm{Sr}_{16} \mathrm{Fe}_{16} \mathrm{O}_{43} \\
\left(\mathrm{SrFeO}_{2.69}\right)\end{array}$ & $\operatorname{mix}$ & 0 & 6 & 0 & $\begin{array}{c}10 \\
(6+4)\end{array}$ & $3(\mathrm{Rh}) / 1(\mathrm{~B})$ \\
\hline 6 & $\begin{array}{l}\mathrm{Sr}_{16} \mathrm{Fe}_{16} \mathrm{O}_{42} \\
\left(\mathrm{SrFeO}_{2.625}\right)\end{array}$ & $\operatorname{mix}$ & 0 & 4 & 0 & $\begin{array}{c}12 \\
(4+8)\end{array}$ & $1(\mathrm{Rh}) / 1(\mathrm{~B})$ \\
\hline 7 & $\begin{array}{l}\mathrm{Sr}_{16} \mathrm{Fe}_{16} \mathrm{O}_{41} \\
(\mathrm{SrFeO} 2.56)\end{array}$ & $\operatorname{mix}$ & 0 & 2 & 0 & $\begin{array}{c}14 \\
(2+12)\end{array}$ & $1(\mathrm{Rh}) / 3(\mathrm{~B})$ \\
\hline 8 & $\begin{array}{l}\mathrm{Sr}_{16} \mathrm{Fe}_{16} \mathrm{O}_{40} \\
(\mathrm{SrFeO} 2.5)\end{array}$ & $8\left(\mathrm{Sr}_{2} \mathrm{Fe}_{2} \mathrm{O}_{5}\right)$ & 0 & 0 & 0 & $\begin{array}{c}16 \\
\left(8 \mathrm{Fe}^{3+}(\mathrm{O})+8 \mathrm{Fe}^{3+}(\mathrm{T})\right)\end{array}$ & Single phase \\
\hline
\end{tabular}

Пр имечание. 1-й столбец - количество вакансий $k$ на 16 перовскитных ячеек; 2-й столбец - новая формула для конфигураций $(k$ вакансий на 16 ячеек); 3-й столбец - старая формула для однофазных конфигураций (одна вакансия на $n$ ячеек); 4-й столбец - количество Fе $4+(\mathrm{O})$ в конфигурации; 5-й столбец - количество $\mathrm{Fe}^{4+}(\mathrm{P})$ в конфигурации; 6-й столбец — количество $\mathrm{Fe}^{3.5+}(\mathrm{O})$ в конфигурации; 7-й столбец - количество $\mathrm{Fe}^{3+}(\mathrm{O})$ в конфигурации; 8-й столбец - соотношение фаз в смеси двух фаз; О — октаэдрическое кислородное окружение, Р - пирамидальное кислородное окружение, T - тетраэдрическое окружение. C - кубическая фаза, Теtra - тетрагональная фаза, Rh - ромбическая фаза, В браунмиллеритная фаза.

всех конфигураций. В эту конфигурацию начинаем добавлять по одной вакансии. Сначала рассмотрим все известные однофазные структуры. В соответствии с этой схемой конфигурацию $\mathrm{Sr}_{8} \mathrm{Fe}_{8} \mathrm{O}_{23}$ надо удвоить, $\mathrm{Sr}_{4} \mathrm{Fe}_{4} \mathrm{O}_{11}$ увеличить в 4 раза, а $\mathrm{Sr}_{2} \mathrm{Fe}_{2} \mathrm{O}_{5}$ увеличить в 8 раз. Для этих конфигураций число вакансий равно 2,4 и 8 на 16 перовскитных ячеек соответственно. Общую формулу для всего набора кислородных окружений в идеальных композициях феррита стронция в зависимости от количества кислородных вакансий можно записать как $\mathrm{SrFeO}_{3-k / 16}$ (или $\mathrm{Sr}_{16} \mathrm{Fe}_{16} \mathrm{O}_{48-k}$ ), где $k$ - количество вакансий на 16 перовскитных ячеек. Таким образом, в эту схему укладываются все однофазные композиции: $k=0$ для $\mathrm{Sr}_{16} \mathrm{Fe}_{16} \mathrm{O}_{48}$ (или $\mathrm{SrFeO}_{3}$ ), $k=2$ для $\mathrm{Sr}_{16} \mathrm{Fe}_{16} \mathrm{O}_{46}$ (или $\mathrm{Sr}_{8} \mathrm{Fe}_{8} \mathrm{O}_{23}$ ), $k=3$ для $\mathrm{Sr}_{16} \mathrm{Fe}_{16} \mathrm{O}_{45}, k=4$ для $\mathrm{Sr}_{16} \mathrm{Fe}_{16} \mathrm{O}_{44}$ (или $\mathrm{Sr}_{4} \mathrm{Fe}_{4} \mathrm{O}_{11}$ ) и $k=8$ для $\mathrm{Sr}_{16} \mathrm{Fe}_{16} \mathrm{O}_{40}$ (или $\mathrm{Sr}_{2} \mathrm{Fe}_{2} \mathrm{O}_{5}$ ) (табл. 2). В табл. 2 представлены все возможные валентные состояния железа с разным кислородным окружением и изменение их количества в зависимости от количества вакансий в 16 перовскитных ячейках. Общая формула из литературы хорошо вписывается в предлагаемую схему.

Главным в данном подходе является то, что, исходя из мёссбауэровских данных, для каждой идеальной однофазной конфигурации получаем набор валентных состояний железа с соответствующим локальным кислородным окружением и их долевое соотношение.
На основании предлагаемой схемы и мёссбауэровских данных по валентным состояниям железа с разным локальным окружением, для однофазных композиций можно расписать количество всех возможных валентных состояний железа с соответствующим локальным кислородным окружением для разного количества вакансий и проследить, как при этом происходит переход из одной структуры в другую (табл. 2). Так, исходя из соотношения вкладов парциальных мёссбауэровских спектров различных локальных окружений атомов $\mathrm{Fe}$, для конфигурации $\mathrm{Sr}_{8} \mathrm{Fe}_{8} \mathrm{O}_{23}$ (в новой схеме $\mathrm{Sr}_{16} \mathrm{Fe}_{16} \mathrm{O}_{46}$, $(k=2))$ соотношение трех локальных окружений атомов железа (рис. $3, a$ ) составляет $4 \mathrm{Fe}^{4+}(\mathrm{O}), 4 \mathrm{Fe}^{4+}(\mathrm{P})$, $8 \mathrm{Fe}^{3.5+}(\mathrm{O})$. Для конфигурации $\mathrm{Sr}_{4} \mathrm{Fe}_{4} \mathrm{O}_{11}$ (в новой формуле $\left.\mathrm{Sr}_{16} \mathrm{Fe}_{16} \mathrm{O}_{44},(k=4)\right)$ соотношение двух локальных окружений атомов железа (рис. $3, b)$ составляет $8 \mathrm{Fe}^{4+}(\mathrm{P})$ и $8 \mathrm{Fe}^{3+}(\mathrm{O})$. Для конфигурации $\mathrm{Sr}_{16} \mathrm{Fe}_{16} \mathrm{O}_{45}(k=3)$ соотношение четырех локальных окружений атомов железа (рис. 3,c) составляет $6 \mathrm{Fe}^{4+}(\mathrm{O}), 2 \mathrm{Fe}^{4+}(\mathrm{P}), 4 \mathrm{Fe}^{3.5+}(\mathrm{O})$ и $4 \mathrm{Fe}^{3+}(\mathrm{O})$. Для конфигурации $\mathrm{Sr}_{2} \mathrm{Fe}_{2} \mathrm{O}_{5}$ (в новой формуле $\left.\mathrm{Sr}_{16} \mathrm{Fe}_{16} \mathrm{O}_{40},(k=8)\right)$ соотношение локальных окружений атомов железа в магнитном состоянии (рис. 3,d) составляет $8 \mathrm{Fe}^{3+}(\mathrm{O}), 8 \mathrm{Fe}^{3+}(\mathrm{T})$.

Наряду с однофазными образцами, серия известных из литературы двухфазных образцов [4,9,11,14,17-19] хорошо укладывается по составу кислорода в предложенную схему для $k=1,5,6,7$. 
Используя данные о локальных окружениях атомов железа в однофазных образцах $(k=0,2,3,4,8)$, можно выстроить картину локальных окружений для оставшихся $k=1,5,6,7$. В зависимости от количества вакансий в конфигурациях, состоящих из 16 перовскитных ячеек, можно достроить последовательность переходов ионов железа из одного валентного состояния в другое и оценить соотношение локальных окружений для двухфазных образцов (табл. 2). Тогда, согласно предлагаемой схеме, для $k=1$ (смесь кубической и тетрагональной фаз $[4,11,17]) \mathrm{Fe}^{4+}$ должен распадаться на три валентных состояния железа с соотношением локальных окружений, указанных в табл. 2. Состав по кислороду для этой конфигурации $\left(\mathrm{SrFeO}_{2.94}\right)$, оцененный из мёссбауэровских данных, хорошо согласуется с экспериментальными данными $[4,11,17]$. Аналогичная ситуация получается для $k=5,6,7$, где существует смесь ромбической и браунмиллеритной фаз (табл. 2). Оцененный состав по кислороду будет $\mathrm{SrFeO}_{2.69}$ для $k=5, \mathrm{SrFeO}_{2.625}$ для $k=6$ и $\mathrm{SrFeO}_{2.56}$ для $k=7$, что хорошо согласуется с литературными экспериментальными данными для $\mathrm{SrFeO}_{2.69}[4,17], \mathrm{SrFeO}_{2.60}$ [18], $\mathrm{SrFeO}_{2.57}$ [4].

Используя предложенную выше схему и мёссбауэровские данные по локальным окружениям, определим соотношение фаз в двухфазных структурах (табл. 2). Согласно литературным данным $[4,11,17]$, для $k=1$ имеем смесь кубической и тетрагональной фаз. Тетрагональная фаза с конфигурацией $\mathrm{Sr}_{8} \mathrm{Fe}_{8} \mathrm{O}_{23}$ имеет 8 ионов $\mathrm{Fe}\left(2 \mathrm{Fe}^{4+}(\mathrm{O}), 2 \mathrm{Fe}^{4+}(\mathrm{P}), 4 \mathrm{Fe}^{3.5+}(\mathrm{O})\right)$. Согласно предлагаемой схемы (табл. 2), общее количество $\mathrm{Fe}^{4+}(\mathrm{O})$ для $k=1$ равно 10. Тогда оставшиеся 8 ионов $\mathrm{Fe}^{4+}(\mathrm{O})$ относятся к кубической фазе $\mathrm{SrFeO}_{3}$, и соотношение фаз для $k=1$ составляет 4/1.

Для $k=5,6,7$ имеем смесь ромбической и браунмиллеритной (брм) фаз. В ромбической фазе с конфигурацией $\mathrm{Sr}_{4} \mathrm{Fe}_{4} \mathrm{O}_{11}$ имеется 4 иона $\mathrm{Fe}\left(2 \mathrm{Fe}^{4+}(\mathrm{P})\right.$ и $\left.2 \mathrm{Fe}^{3+}(\mathrm{O})\right)$, тогда согласно схеме, для $k=5$ конфигурация и соответственно количество ионов Fе увеличивается в три раза. Общее количество $\mathrm{Fe}^{3+}(\mathrm{O})$ для $k=5$ в этой схеме равно 10. На браунмиллеритую фазу остается 4 иона $\mathrm{Fe}^{3+}$. Браунмиллеритная фаза с конфигурацией $\mathrm{Sr}_{2} \mathrm{Fe}_{2} \mathrm{O}_{5}$ имеет 2 иона $\mathrm{Fe}^{3+}$ (с октаэдрическим $\left(\mathrm{Fe}^{3+}(\mathrm{O})\right.$ и тетраэдрическим $\mathrm{Fe}^{3+}(\mathrm{T})$ кислородным окружением). В итоге соотношение фаз для $k=5$ составляет 3/1. Для $k=6$ конфигурация $\mathrm{Sr}_{4} \mathrm{Fe}_{4} \mathrm{O}_{11}$ и соответственно количество $\mathrm{Fe}$ удваивается. Из 12 общих ионов $\mathrm{Fe}^{3+}$ остается 8 на браунмиллеритую фазу, и соотношение фаз составляет 1/1. Выше приведены результаты исследованной в данной работе смеси браунмиллеритной и ромбической фаз. Оцененное из экспериментальных данных соотношение фаз хорошо укладывается в предлагаемую схему для $k=6$. Для $k=7$ из 14 ионов $\mathrm{Fe}^{3+}$ остается 12 на браунмиллеритую фазу, и соотношение фаз составляет $1 / 3$ (табл. 2). Данные табл. 2 для наглядности приведены в графическом виде на рис. 4. Приведенные в таблице дискретные структурные композиции, рассчитанные с шагом в одну вакансию $(k=1)$, являются здесь опорными точками в количественных зависимостях для кислородных окружений. Используя графики, можно оценить их соотношения и для промежуточных случаев.

Таким образом, получается единая картина последовательных переходов железа из одного валентного состояния в другое при добавлении вакансий и всех возможных его кислородных окружений независимо от количества фаз, получаемая из мёссбауэровской спектроскопии. Каждому структурному состоянию (одноили двухфазному) соответствует определенный набор валентных состояний железа с определенным локальным кислородным окружением. Зная соотношение валентных состояний $\mathrm{Fe}$ для однофазных идеальных конфигураций, можно оценить соотношение фаз для двухфазных композиций (табл. 2).

\section{4. Заключение}

Проведены структурные исследования браунмиллеритной фазы для образцов, отожженных в вакууме при разных температурах. Установлено, что все возможные валентные состояния железа в кислородно-дефицитном оксиде $\mathrm{SrFeO}_{3-\delta}$ (одно- и двухфазных системах) однозначно определяются кислородно-вакансионным окружением.

Выстроена последовательность переходов $\mathrm{Fe}$ из одного валентного состояния в другое при добавлении вакансий.

Предлагаемая схема является более общим случаем для описания всех известных соединений $\mathrm{SrFeO}_{3-\delta}$ и в нее хорошо вписывается рассматриваемая в литературе формула для однофазных структур. Существующие в литературе данные по одно- и двухфазным составам хорошо укладываются в эту схему.

С помощью данной схемы для идеальных композиций можно оценивать соотношение валентных состояний железа и соотношение фаз в экспериментально получаемых двух- и однофазных системах с разным содержанием кислорода и разным фазовым составом.

\section{Благодарности}

Авторы выражают глубокую благодарность В.В. Кведеру и Н.В. Барковскому за проявленный интерес и полезные обсуждения полученных результатов.

\section{Финансирование работы}

Работа выполнена в рамках государственного задания Министерства образования и науки РФ.

\section{Конфликт интересов}

Авторы заявляют, что у них нет конфликта интересов. 


\section{Список литературы}

[1] B. Schmid, G. Rupp, J. Fleig. Phys. Chem. Chem. Phys. 20, 12016 (2018).

[2] D. Gryaznov, R. Merkle, E. Kotomin, J. Maier. J. Mater. Chem. A 4, 13093 (2016).

[3] G. Dong, H. Fan, H. Tian, J. Fang, Q. Li. RSC Adv. 5, 29618 (2015).

[4] Y. Takeda, K. Kanno, T. Takada, O. Yamamoto, M. Takano, N. Nakayama, Y. Bando. J. Solid State Chem. 63, 237 (1986).

[5] M. Takano, T. Okita, M. Nakayama, Y. Bando, Y. Takeda, O. Yamamoto, J.B.J. Goodenough. J. Solid State Chem. 73, 140 (1988).

[6] S.H. Lee, T.W. Frawley, C.H. Yao, Y.C. Lai, Chao-Hung Du, P.D. Hatton, M.J. Wang, F.C. Chou, D.J. Huang. New J. Phys. 18, 093033 (2016).

[7] E. Heifets, E.A. Kotomin, A.A. Bagaturyants. J. Maier. Phys. Chem. Chem. Phys. 21, 3918 (2019).

[8] A. Maity, R. Dutta, B. Penkala, M. Ceretti, A. Letrouit-Lebranchu, D. Chernyshov, A. Perichon, A. Piovano, A. Bossak, M. Meven, W. Paulus. J. Phys. D 48, 504004 (2015).

[9] S.H. Hsieh, R.S. Solanki1, Y.F. Wang, Y.C. Shao, S.H. Lee, C.H. Yao, C.H. Du, H.T. Wang, J.W. Chiou, Y.Y. Chin, H.M. Tsai, J.-L. Chen, C.W. Pao, C.-M. Cheng, W.-C. Chen, H.J. Lin, J.F. Lee, F.C. Chou, W.F. Pong. Sci. Rep. (2017). On line.

[10] M. Ceretti, A. Piovano, A. Cousson, T. Berthier, M. Meven, G. Agostini, J. Schefer, O. Hernandez, O. Lamberti, W. Paulus. Cryst. Eng. Commun. 14, 5771 (2012).

[11] P. Adler, J. Strempfer, B. Keimer, V. Duppel, F. Phillipp. J. Phys. Chem. Solids 68, 73 (2007).

[12] S. Schott, Zh. Jia, A.K. Schaper, V. Thangadurai, W. Weppner, P. Schmid- Beurmann. Phys. Status Solidi A 202, 12, 2330 (2005).

[13] J.P. Hodges, S. Short, J.D. Jorgensen, X. Xiong, B. Dabrowski, S.M. Mini, C.W. Kimball. J. Solid State Chem. 151, 190 (2000).

[14] H. Falcón, J.A. Barbero, J.A. Alonso, M.J. Martínez-Lope, J.L.G. Fierro. Chem. Mater. 14, 2325 (2002).

[15] M. Schmidt, M. Hofmann, S.J. Campbell. J. Solid State Chem. 151, 190 (2000).

[16] M. Schmidt, M. Hofmann, S.J. Campbell. J. Phys.: Condens. Matter 15, 8691 (2003).

[17] P. Adler, A. Lebon, V. Damljanovic, C. Ulrich, C. Bernhard, A.V. Boris, A. Maljuk, C.T. Lin, B. Keimer. Phys. Rev. B 73, 094451 (2006).

[18] P.K. Gallagher, J.B. MacChesney, D.N.E. Buchanan. J. Chem. Phys. 41, 8, 2429 (1964).

[19] P. Manimuthu, R. Murugaraj, C. Venkateswaran. Phys. Lett. А 378, 2725 (2014).

[20] В.Д. Седых, О.Г. Рыбченко, А.Н. Некрасов, И.Е. Конева, В.И. Кулаков. ФТТ 61, 6, 1162 (2019).

[21] M. Schmidt, S.J. Campbell. J. Solid State Chem. 156, 292 (2001)

[22] J.E. Auckett, A.J. Studer, N. Sharma, C.D. Ling. Solid State Ion. 225, 432 (2012).

[23] H. D'Hondt, A.M. Abakumov, J. Hadermann, A.S. Kalyuzhnaya, M.G. Rozova, E.V. Antipov, G. Van Tendeloo. Chem. Mater. 20, 7188 (2008).

[24] V. Sedykh, F.E. Fujita, I.S. Smirnova, A.V. Dubovitskii, B.Zh. Narymbetov, V.Sh. Shekhtman. Jpn. J. Appl. Phys. 34, 4033 (1995).

Редактор Ю.Э. Китаев 